\title{
An Overview of Reconfigurable Antennas for Wireless Body Area Networks and Possible Future Prospects
}

\author{
Hafiz Suliman Munawar
}

University of New South Wales (UNSW) Sydney, Australia

Received: 06 January 2020; Accepted: 08 March 2020; Published: 08 April 2020

\begin{abstract}
The use of antennas in various fields and applications is gaining much importance because of their growing advancements and numerous facilities. Due to their vast advantages, now wearable antennas are used in fields of personal healthcare, entertainment, military and many others. For the fabrication of wearable antennas, many aspects need special consideration in order to manufacture a safe system for the user. As the applications of wireless body centric communication with antennas has a great variability, so enhancements are made in the systems by integrating reconfigurable antennas in it. These antennas work better than the single installed antennas because of their adjustable parameters thus increasing the performance of whole system. This paper provides an overview of the existing antennas and their applications, in order to better understand ways in which the limitations associated with wearable antennas could be overcome in the future. Certain design challenges which occur during the fabrication of wearable antennas like safety, cost and tolerance in addition to the recent advancements in this field are discussed. Designing a particular antenna for body area network mainly depends upon the application of the user and the place of installation. To build a review about the reconfigurable antenna for body centric communication, 15 papers from the last 6 years are consulted which fall under the domain of wearable antennas. There is still a margin of improvement in this field and better technologies will be introduced in coming years which include precision medicine.
\end{abstract}

Index Terms: Body-centric communication system, pattern reconfigurable antenna, wearable antenna, onbody, flexible, reconfigurable frequency.

(C) 2020 Published by MECS Publisher. Selection and/or peer review under responsibility of the Research Association of Modern Education and Computer Science

\footnotetext{
* Corresponding author

E-mail address: h.munawar@unsw.edu.au
} 


\section{Introduction}

Wearable antennas are specifically designed to provide useful facilities with the ease of use to the user. One such example of these antennas is smartwatches which gained huge popularity in recent years, providing an easy means of communication along with various other features. With growing boom in videography, GoPro cameras are now widely used which have the $\mathrm{Wi}-\mathrm{Fi}$ and Bluetooth facility in it and can be easily strapped to the user body for footage. As these antennas have a huge market and applications so with slight modification in parameters, they can render useful products. Keeping these prospects in mind, reconfigurable antennas are integrated in body area networks to further develop this field.

Reconfigurable antennas proved to be a subject of many researches and became a part of many smart and adaptive systems because of their capability to rearrange one or more of the parameters like frequency or polarization. These antennas can perform various operations on a single hardware using different frequency bands which makes them compact, cost efficient and flexible. They are intended to reduce the size and interference in the system while increasing its performance [1]. Various capabilities like good isolation, less processing and minimized volume make them suitable for use in several wireless applications. Reconfigurable antennas can be achieved by altering frequency, bandwidth, pattern, polarization, radiation and several other methods.

Keeping in view of the advancements made in wireless communication, this technology has been adequately applied in many biomedical, military, personal health and industrial applications. Several advances are made in extending the wireless technology to body area as human body serves as an inviting host for the wireless signal [2]. Body centric wireless communication systems (BCWS) works in two modes, one is on body communication and other is off body communication. For the first mode of communication, sensors collect the data and communicate it over the body they are called body worn antennas, textile antennas or wearable antennas. As human body has transmission losses so antennas having normal polarization with the body are used. For off-body communication, broadside radiation pattern antennas are used [3]. In fields of medical and health care, BCWS has proved its value with its use in Electrocardiogram (ECG), blood flow, Electroencephalography (EEG) as they help in patient monitoring and healthcare of elderly [4]. Not only these advancements are used on earth, but they have vast applications in space also. Communication links with the astronauts can be built using off-body communication network.

In order to use human body as antenna, various wearable antennas are developed which include flexible antenna for wearing spectacles applications, radiation antenna with the use of Bluetooth headset, repeater antenna for body sensors and shell antennas for hearing instruments. Most important part of any wearable antenna is that it should be made of suitable material which is safe for the wearer. Latest advancements use the placement of the antenna in the zipper of a handbag which is modified version of jacket zipper antenna but provides better performance [5].

\section{A. Working of BCWS}

For a smooth communication system, two links are formed which are inductive link and the far-field radio frequency link. Inductive link is beneficial for the communication in near fields as they adopt coils to transfer the data over short distances. Far- field link can perform the communication over long distances, it also has multiple user access along with a higher rate of transmission. The antennas which can be implanted in the body works as the transmitting antenna and the receiving antenna is operated externally. This increases the complexity of the system because of multipath effect and polarization mismatching. In order to minimize these effects, polarizable reconfigurable antennas are applied which avoid multipath effect and have polarization coding capability [6].

This review paper is organized as follows: section 2 provides a review of some of the existing technologies in wearable antennas in addition to a comparison table which briefly discusses some applications and limitations of reconfigurable wearable antennas. Section 3 covers certain design challenges and recent advancements in this field. Section 4 concludes the paper. 


\section{Methodology}

Reconfigurable antennas are being used in wireless body area networks (WBAN) to enhance the existing technologies of personal healthcare, military and entertainment. Most important part of any wearable antenna is that it should not be an obstacle in the movement of the wearer. The system should be light in weight and compact in size for the ease of carrying otherwise it will create problems for the wearer. To form a review of the methods and technologies that serve in the field of wearable antennas, it is much needed to have a background knowledge of the already implemented technologies.

As the concept of reconfigurable antennas for wearable technology is a vast field so it is needed to set the boundary of this review. This review contains papers published in the conferences and journals from International Electrical and Electronics Engineers (IEEE) and Institution of Electrical Engineering (IET) for past 6 years in order to have knowledge of the recent technologies.

This section discusses some of the existing technologies for wearable antennas along with the limitations of these systems in order to find a way which help in overcoming these limitations for future purposes.

\subsection{Pattern reconfigurable antenna for on body communication}

In order to cater for the transmission losses due to human body, monopole and surface wave antennas are used because they can launch creeping waves effectively for the node located in non- line of sight. Pattern reconfigurable antennas are directional in nature as they can change their beam direction, depending upon the requirements of the system. Directional antennas also have better signal sensitivity and long-range communication [7].

$\mathrm{Li}$ et al [2] presented a reconfigurable antenna which has maximum radiation of $\pm 60^{\circ}$ in elevation plane along with switchable tilted beam radiation. Frequency of operation for this antenna was $2.4 \mathrm{GHz}$ and contained Yagi-like structures as the radiating element. Ground plane was provided by a conducting vias and rectangular metallic patches which provide a high impedance surface (HIS). This surface obtains the desired phase of reflection and dispersion diagram. Antenna performance was observed for free space and on a biological tissue to make it compatible for the human body. With the use of diffraction in HIS, the antenna showed radiation with tilted angle. The tilt of radiation for free space was $60^{\circ}$ which is significantly large and can be used for on body communication applications.

\subsection{Reconfigurable antenna for $2.45 / 5 \mathrm{GHz}$ frequency band}

Previously, many antennas for body area networks used full ground planes and electromagnetic couple feed. Abbas et al [4] used a partial ground plane which is easily fabricated because of its ability to avoid narrow gaps between strips. They used both, partial and full ground planes to investigate which one works better in various circumstances. The antennas were tested for an Industrial, Scientific and Medical (ISM) band at $2.45 \mathrm{GHz}$ and Wireless Local Area Network at $5 \mathrm{GHz}$.

The basic parameter which was investigated in this experiment was the sensitivity of both the antennas towards the gap between human body and antenna. Results showed that antenna with full ground was suitable for narrow band and long-range operations which requires the gap between human body and antenna to be varying, whereas the partial ground plane worked best where more bandwidth is a requirement. The antennas were smaller in size, so they can easily be worn. Proposed antennas provided wide radiation pattern which supported their use in on-body communication.

\subsection{Polarization reconfigurable multi-slot antenna}


Antennas with properties of pattern configurability are used extensively for BWCS in order to avoid multipath effect. Diversity in polarization can be achieved by designing own reconfigurable radiators or by adopting the already reconfigurable feeder [8]. Lin et al [6] presented four linear polarizations at the angles of $0^{\circ},+45^{\circ}, 90^{\circ}$ and $-45^{\circ}$. For the proposed design of the antenna, multi-slot radiators were placed at the substrate. PIN diodes were used for switching purposes as RF switches and biasing voltages were provided by using DC lines and posts. Balanced differentials sources were needed for exciting the multi-slot radiators which was done by designing tapered Balun connectors.

Furthermore, radiators were arranged in specific manner and slots which were controlled by the ON/OFF status of the diode. Results obtained from the designed antenna showed that it worked well in all modes of polarization keeping high gain and consistency and they can effectively evade the issue of polarization mismatching. Good radiation patterns for broadside were obtained for all the modes which made it useful in BCWS applications.

\subsection{Textile integrated wearable antennas}

As the earlier developed antennas needed to be in contact with the body which can cause discomfort in some scenarios, so advancements were made to integrate the antennas on the garments in order to facilitate the wearer. For this purpose, the antennas need to be flexible enough so that they can easily be integrated with clothes. Most of the configurable frequency antennas previously designed were used on micro strip which cannot be used on body [9].

Shakhirul et al [7] presented the design for reconfigurable frequency antennas with usage of textile material in addition to circular polarization. Radiating element in the described antenna was Shield it Super whereas the thickness of the fabric was $1.7 \mathrm{~mm}$. Three switches were placed, out of which first and third were placed at right and left of the slotted, whereas the middle switch was placed at the middle of the slotted. DC biasing and separation of the switches was done using four narrow slots. Results obtained from the designed antenna showed that the modification of slotted at the ground facilitated in achieving different operating frequencies. Good circular polarization was also obtained which made this antenna useful for wireless communication as well as for many GPS applications.

\subsection{Watchband antenna for wearable systems}

As the previously discussed antenna for the textile provides good performance and results but it required certain specifications to make it compatible for the application purpose. New advancements were still needed to be made in order to have better and comfortable devices for on-body communication systems. Considering these needs, Li et al [8] gave the proposal of the antenna which used the metal wrist watchband. For experimentation purposes, TISSOT watch was selected and results were demonstrated. Watch dial had a fixed top with a metallic frame and glass lid along with air spaces around the band.

Reflection coefficients depend greatly upon the feeding location, and the excitation was produced at the right side of the dial and at the front and back. As the posture of arm changes at different time instants, so a broader main lobe was expected. Different hand postures were deployed, and fairly good performance was observed which proved the feasibility of the designed system and validated the novel concept for wearable applications [10].

\subsection{Zipper antenna for BCWS}

As the body centric communication can also be done by using on-body as well as off-body antenna. Many of the on-body systems were deployed and proved good performance but they need to be in contact with the body which is not possible in all cases. In military or secret surveillance, off-body antennas have greater importance [11]. One kind of off-body antenna is a handbag zipper antenna. Li et al [4] gave the design of such handbag 
zipper which was previously developed for jacket zipper, but the performance of jacket zipper varied depending on whether the zip is closed or open.

Handbag zipper was designed to make an advancement without the requirement of any additional fabrication as it used the already present metal of the zipper. Radiation pattern for closed, open, quarterly or half open were observed which showed the change in the direction of main radiation pattern. Furthermore, the impact of width of zipper teeth on radiation was also studied. Results obtained from the simulations of the whole system showed that for frequency bands, special impedance structures could be designed. The size of this antenna was relatively bigger than common wearable antennas which increased the gain and performance. By changing the feeding location, this antenna could work in different frequency bands also it was a cost-efficient system because of no special fabrication requirement [4].

\subsection{Waveguide frequency reconfigurable antenna}

The designed system presented by Khan et al [12] worked as a reconfigurable antenna by using Dielectric Resonator antenna (DRA) and a coplanar waveguide. The proposed design could reconfigure frequencies at four bands which was done by using two switches that performed three switching operation. For every switching operation, impedance bandwidth is changed. The volume of the complete system was very small, which made it even useful for wearable technologies.

Table 1. Types of Antenna, their applications and limitations

\begin{tabular}{|c|c|c|c|}
\hline Antenna Types & Communication mode & Applications & Limitations \\
\hline $\begin{array}{l}\text { Pattern } \\
\text { reconfigurable } \\
\text { antenna }\end{array}$ & $\begin{array}{l}\text { On body } \\
\text { communication network. }\end{array}$ & $\begin{array}{l}\text { Wireless communication, } \\
\text { Surveillance, Tracking. }\end{array}$ & $\begin{array}{l}\text { Difficult to vary radiation } \\
\text { pattern below } 1 \mathrm{GHz} \text {. }\end{array}$ \\
\hline $\begin{array}{l}\text { Polarization } \\
\text { reconfigurable } \\
\text { antenna }\end{array}$ & $\begin{array}{l}\text { On body } \\
\text { communication network. }\end{array}$ & $\begin{array}{l}\text { Biomedical applications } \\
\text { (insulin pumps, blood pressure } \\
\text { monitor). }\end{array}$ & Complex switching. \\
\hline $\begin{array}{l}\text { Textile integrated } \\
\text { antennas }\end{array}$ & $\begin{array}{l}\text { On body } \\
\text { communication network. }\end{array}$ & $\begin{array}{l}\text { Smart clothes for firefighters, } \\
\text { GPS loaded vests. }\end{array}$ & $\begin{array}{l}\text { Need special fabrication } \\
\text { of cloth that has } \\
\text { conducting abilities. }\end{array}$ \\
\hline Watchband antennas & $\begin{array}{l}\text { On body } \\
\text { communication network. }\end{array}$ & $\begin{array}{l}\text { Secret operators, Security, } \\
\text { Smart watches. }\end{array}$ & $\begin{array}{l}\text { Matching performance } \\
\text { position. }\end{array}$ \\
\hline Zipper antennas & $\begin{array}{l}\text { Off body communication } \\
\text { network. }\end{array}$ & $\begin{array}{l}\text { Environment } \\
\text { monitoring, Communication, } \\
\text { Smart cities. }\end{array}$ & $\begin{array}{l}\text { Movement and distance } \\
\text { between the bag and } \\
\text { human will cause } \\
\text { performance reduction. }\end{array}$ \\
\hline
\end{tabular}

\subsection{WBAN for aeronautics}

As space exploration is advancing with great pace and humans are travelling to outer space, it was very much needed to develop a system that can form a communication link in space. Przybylski et al [10] designed a system to communicate with humans travelling to space. The designed system was a kind of off-body network that had a sensor network, transmitter and antenna in addition to second subsystem which was an android device. Proposed system was deployed and tested in a lab using ECG sensors and Nexus 7.

In order to have a comprehensive knowledge of reconfigurable antennas and their applications, a comparative analysis of them is conducted which is shown in Table 1. This table provides an overview of the different types of antennas based on their mode of communication and the existing limitations. This helps in identifying the key points of each type of antenna and highlights the areas that still need to be covered.

After studying different type of body centric antennas, it shows that every antenna has a specialty of its own which makes it different from others. Some of the on-body antennas work best in places that involve a lot of movement because of their flexible nature. At the same time their fabrication may require complex system or special arrangement. Choice of any antenna is mainly dependent upon the application and design constraints of 
the user. For example, the use of wearable antennas for detection of medical conditions or tracking health in the remote areas through satellites would require specific technology aids and ensuring that the network is set up to match the frequencies of the medical equipment and vice versa $[9,13]$.

\section{Design challenges and advancements}

With the current progress and wide range usage of wearable electronics it is much needed to use the electronics in wireless communication. For this purpose, various wearable antennas are developed which include health watch, smart watch and Nike+ sensors. This field is still growing rapidly in order to enhance the facilities and build a smooth communication link. In order to form a wearable gadget which can perform the tasks of communication, it is much needed to have a fine and high-quality fabrication process without being very costly. Some of the key challenges which occur during the fabrication of a wearable antenna are cost, flexibility, tolerance and weight.

One type of body-worn antennas is thin film antennas, they work by depositing a metal upon a flexible substrate. Screen printing is the most efficient amongst them in terms of cost and implementation. Photolithography is also used extensively in the formation of flexible antennas. Apart from these methods, various other ways to realize a thin film wearable antenna are also used which include inkjet printing and manual deposition. The problem with thin film antennas is their sensitiveness towards extreme conditions and mechanical stress [14].

As discussed earlier, body worn antennas can be sometimes uncomfortable that's why technology has switched to textile integrated antennas. For fabric integration it is needed to deposit some conductive material on the fabric as well which is done by several methods.

One of the original deposition methods is the use of flexible copper tape which easily sticks on the fabric. This method is easy and cost effective, but they are not robust and have the issue of delamination. Another method is the use of E-threads which are conductive in nature and have great resistance against washing and mechanical stresses [15].

\section{Conclusion and future enhancements}

Different method for the implementation of the wearable antennas for the purpose of communication are studied for this review. It discusses different types of the reconfigurable antenna along with brief method of working. Results from different reconfigurable antennas are presented, in order to have clear knowledge of their implementation. Some of the on-body and off-body communication networks are discussed which tells that there has been done great research in the field of integrating external sources in the body-centric network for more efficient and better performance.

Design of the specific body-worn or textile integrated antenna is the most crucial and important task. For body-worn antenna, the selected material should be able to perform in conductive as well as nonconductive parts while remaining in the safe zones for the user. In textile integrated antenna, there can arise issues of wet clothes or harsh external environment. These factors need special consideration before designing a costefficient, light weight and compact antenna for the process of communication in body area networks. Thorough researches done for enhancing the experience with wearable technology prove that it will keep growing and provide many new facilities. Extending these fields of antenna technology in healthcare will provide better health experience in coming years which include precision medicine and treatments. Especially the provision of healthcare support in under-privileged regions or remote areas can be highly benefited if the technology is made more feasible and cost effective. Hence, there are various avenues regarding antennas that remain to be explored and there is a lot of potential within this area of research which can be addressed by future studies. 


\section{References}

[1] Salleh, S.M., Jusoh, M., Ismail, A.H., Kamarudin, M.R., Nobles, P., Rahim, M.K.A., Sabapathy, T., Osman, M.N., Jais, M.I. and Soh, P.J., 2017. Textile antenna with simultaneous frequency and polarization reconfiguration for WBAN. IEEE Access, 6, pp.7350-7358.

[2] Li, M., Xiao, S.Q. and Wang, B.Z., 2013, December. Pattern-reconfigurable antenna for on-body communication. In 2013 IEEE MTT-S International Microwave Workshop Series on RF and Wireless Technologies for Biomedical and Healthcare Applications (IMWS-BIO) (pp. 1-3). IEEE.

[3] Saeed, S.M., Balanis, C.A., Birtcher, C.R., Durgun, A.C. and Shaman, H.N., 2017. Wearable flexible reconfigurable antenna integrated with artificial magnetic conductor. IEEE Antennas and Wireless Propagation Letters, 16, pp.2396-2399].

[4] Abbas, S.M., Ranga, Y. and Esselle, K.P., 2015, August. Reconfigurable antenna options for 2.45/5 GHz wireless body area networks in healthcare applications. In 2015 37th Annual International Conference of the IEEE Engineering in Medicine and Biology Society (EMBC) (pp. 5465-5468). IEEE.

[5] Li, G., Huang, Y., Gao, G., Wei, X., Tian, Z. and Bian, L.A., 2017. A handbag zipper antenna for the applications of body-centric wireless communications and Internet of Things. IEEE Transactions on Antennas and Propagation, 65(10), pp.5137-5146.

[6] Lin, W. and Wong, H., 2016, May. Polarization reconfigurable multi-slot antenna for body-centric wireless communication system. In 2016 IEEE International Workshop on Electromagnetics: Applications and Student Innovation Competition (iWEM) (pp. 1-3). IEEE.

[7] Shakhirul, M.S., Jusoh, M., Ismail, A.H., Kamarudin, M.R., Rahim, H.A. and Sabapathy, T., 2016, April. Reconfigurable frequency with circular polarization for on-body wearable textile antenna. In 2016 10th European Conference on Antennas and Propagation (EuCAP) (pp. 1-4). IEEE.

[8] Li, G., Gao, G., Liu, W., Su, J. and Bai, Y., 2017, July. An on-body watchband antenna for the applications of wearable systems. In 2017 IEEE International Symposium on Antennas and Propagation \& USNC/URSI National Radio Science Meeting (pp. 585-586). IEEE.

[9] Munawar, H.S., AAwan, A.A., Khalid, U., Munawar, S. and Maqsood, A., 2017. Revolutionizing Telemedicine by Instilling H. 265. International Journal of Image, Graphics \& Signal Processing, 9(5).

[10] Przybylski, T., Froehle, P., McDonald, C., Mirzaee, M., Noghanian, S. and Fazel-Rezai, R., 2015, May. Wearable wireless body area network for aeronautical applications. In 2015 IEEE International Conference on Electro/Information Technology (EIT) (pp. 563-568). IEEE.

[11] Alharbi, S., Shubair, R.M. and Kiourti, A., 2018. Flexible antennas for wearable applications: Recent advances and design challenges.

[12] Khan, S., Ali, H., Nasir, J., Khan, R., Ramenah, H. and Tanougast, C., 2018, September. A Coplanar Waveguide Wide Band Frequency Reconfigurable Dielectric Resonator Antenna for Wimax/WLAN/WBAN Applications. In 2018 International Conference on Signals and Electronic Systems (ICSES) (pp. 191-195). IEEE.

[13] Munawar, H.S., Awan, A.A., Maqsood, A. and Khalid, U., REINVENTING RADIOLOGY IN MODERN ERA.

[14] Casula, G.A., Montisci, G., Valente, G. and Gatto, G., 2018. A robust printed antenna for UHF wearable applications. IEEE Transactions on Antennas and Propagation, 66(8), pp.4337-4342.

[15] Gao, G.P., Hu, B., Wang, S.F. and Yang, C., 2018. Wearable circular ring slot antenna with EBG structure for wireless body area network. IEEE Antennas and Wireless Propagation Letters, 17(3), pp.434-437. 


\section{Author's Profile}

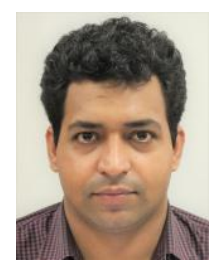

Hafiz Suliman Munawar is a PhD student at the University of New South Wales (UNSW), Australia. He is a multi-disciplinary researcher with experience in machine learning, disaster management and artificial intelligence. Hafiz has several international publications in various journals and conferences and has actively been working on disaster management

How to cite this paper: Hafiz Suliman Munawar, " An Overview of Reconfigurable Antennas for Wireless Body Area Networks and Possible Future Prospects ", International Journal of Wireless and Microwave Technologies(IJWMT), Vol.10, No.2, pp. 1-8, 2020.DOI: 10.5815/ijwmt.2020.02.01 